Address for Correspondence: Dr. Vishal Sehgal, University of Tennessee Health Science Center. Memphis, Tennessee 38163, USA. Email: vsehgal@uthsc.edu

\begin{tabular}{|c|}
\hline Access this article online \\
\hline $\begin{array}{l}\text { Website: } \\
\text { www.intern-med.com }\end{array}$ \\
\hline $\begin{array}{l}\text { DOI: } \\
\text { 10.1515/jtim-2017-0039 }\end{array}$ \\
\hline Quick Response Code: \\
\hline 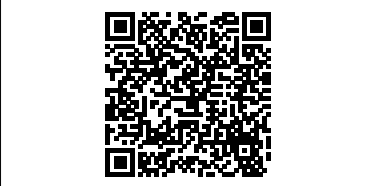 \\
\hline
\end{tabular}

\title{
Urgent need to define Pretreatment predictors of immune check point inhibitors related endocrinopathies: A case report and review of literature
}

\author{
Vishal Sehgal, Richard Childress \\ University of Tennessee Health Science Center, Memphis, Tennessee 38163, USA
}

\section{ABSTRACT}

Immune check point inhibitors have revolutionized the treatment of metastatic malignancies. They are a promising area in oncology and more drugs are likely to be available in the coming years. Along with the promise of better response oncologically, there is an increased incidence of endocrinopathies related to autoimmunity. This case report illustrates the dramatic development of hypothyroidism in a patient with underlying subclinical hyperthyroidism. It also suggests the potential pretreatment predictors of endocrinopathies related to these immune check point inhibitors.

Key words: immune check point inhibitors, endocrinopathies, metastatic malignancy -

\section{INTRODUCTION}

Immune check point inhibitors are a new class of monoclonal antibodies which have shown excellent results in the treatment of metastatic malignancies. But they are what we call as broad spectrum endocrine poisons. It is very important that these endocrinopathies are not missed and are appropriately treated. This would not only reduce the cost, but also improve the morbidity and mortality associated with the use of immune check point inhibitors.

\section{CASE REPORT}

The patient was a 65 -year-old Caucasian male admitted to the hospital for suspected pneumonia related sepsis. He had been feeling weak and lethargic for days. His recent history was significant for squamous cell lung cancer. He had received 6 cycles of chemotherapy with carboplatin/paclitaxel. Two months prior to the presentation, his thyroid function tests (TFT) were consistent with the subclinical hyperthyroidism. His thyroid stimulating hormone (TSH) was $<0.015 \mu \mathrm{IU} / \mathrm{mL}$ and his free T4 was $1.24 \mathrm{ng} / \mathrm{dL}$. On admission, his TSH was $>100 \mu \mathrm{IU} / \mathrm{mL}$ and his free T4 had dropped to $0.28 \mathrm{ng} / \mathrm{dL}$. Endocrinology was consulted for the abnormal thyroid function tests (TFT) (Figures 1 and 2). The acute rise in TSH and acute fall in free T4 level was very striking. On further questioning, the patient confided that he had been getting new medicine at his oncologist's office. A call to the oncologist's office confirmed that he got the first dose of immune check point inhibitor (nivolumab $240 \mathrm{mg}$ ) 2 weeks back. He was then started on low dose thyroid hormone replacement therapy (THRT) with levothyroxine at $50 \mathrm{mcg}$ daily. Further work in the hospital was not indicative of sepsis. His antibiotics were stopped, and he was subsequently discharged from the hospital.

\section{DISCUSSION}

\section{Metastatic malignancies and immune modulation}

The era of immune modulation in the treatment of cancer is relatively recent and trials are underway to see their effect in a range of malignancies. The European Society for Medical Oncology in Vienna highlighted the role of immunotherapy in advanced kidney and lung cancers. ${ }^{[1,2]}$ 


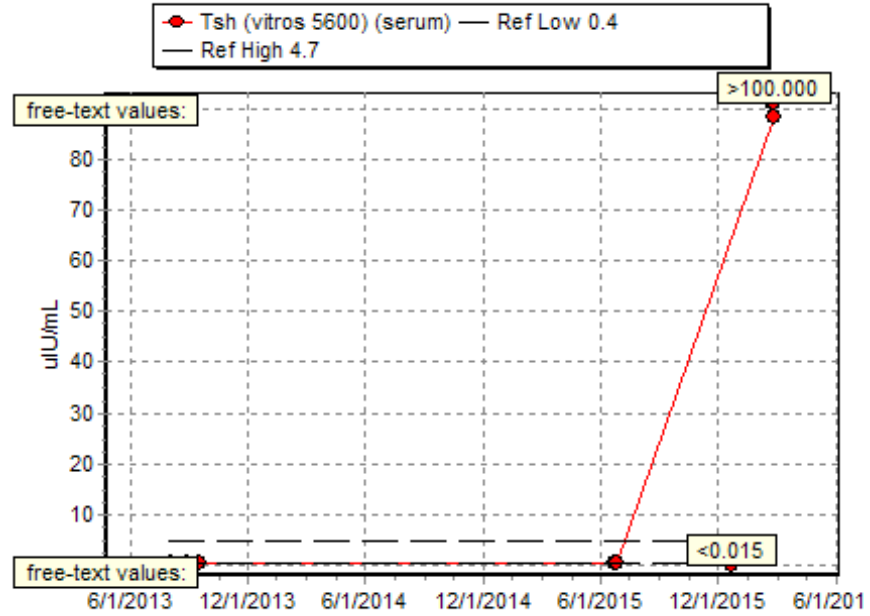

Figure 1: Dramatic rise in thyroid stimulating hormone (TSH) 8 weeks after starting nivolumab

Immune checkpoint inhibitors are also showing promising results in urological cancers. ${ }^{[3-5]}$ Currently, they are the primary treatment modality for the treatment of metastatic melanoma and non-small cell lung cancer. They have been used solo and in combination.

The most widely used immune check point inhibitors include the inhibitors of cytotoxic- T lymphocyte associated protein-4 (CTLA-4) and programmed death 1(PD-1). These proteins allow tumors to evade the immune system. The CTLA-4 is thought to suppress the immune response early and acts principally in lymph nodes. PD- 1 is a receptor seen mostly in peripheral tissues and is expressed

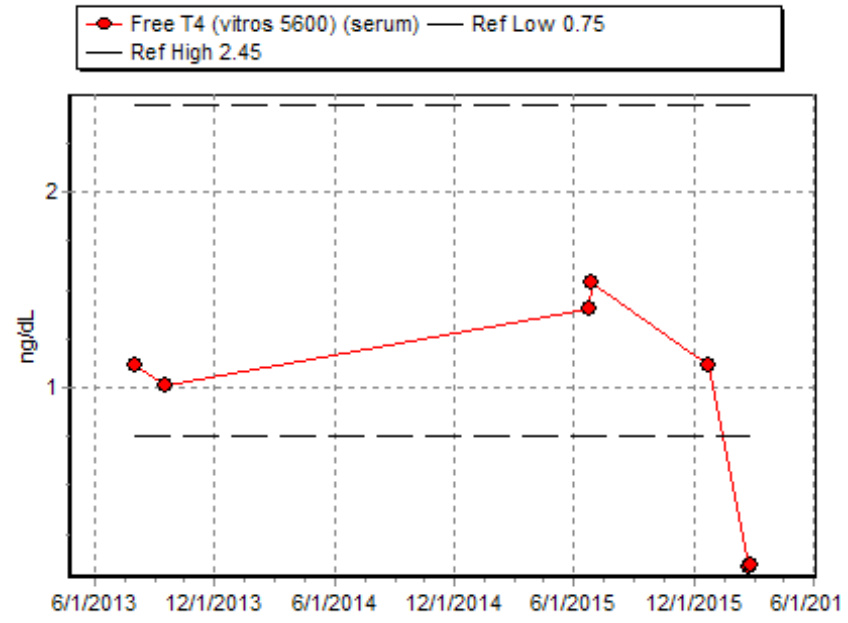

Figure 2: Dramatic fall in freeT4 8 weeks after starting nivolumab

on $\mathrm{T}$ and $\mathrm{B}$ cells, and Natural killer cells. Like CTLA-4, it is a negative regulator of the immune system. It binds to PD-1 ligand, which is expressed in many tumor cells and hematopoietic cells. The interaction of PD-1 with PD-1 ligands blocks the expression of T effector cells. The PD-1 and PD-1 ligands are upregulated by cytokines like IL-2 in the same manner it upregulates CTLA-4 expression. Monoclonal antibodies of the IgG4 class could potentially prevent the interaction of PD-1 receptors on immune cells and PD-1 ligands on tumor cells and hematopoietic cells (Figure 3). Inhibition of these 2 pathways leads to an increased activation of the immune system, which may be used against the cancer cells. s, $^{[6]}$

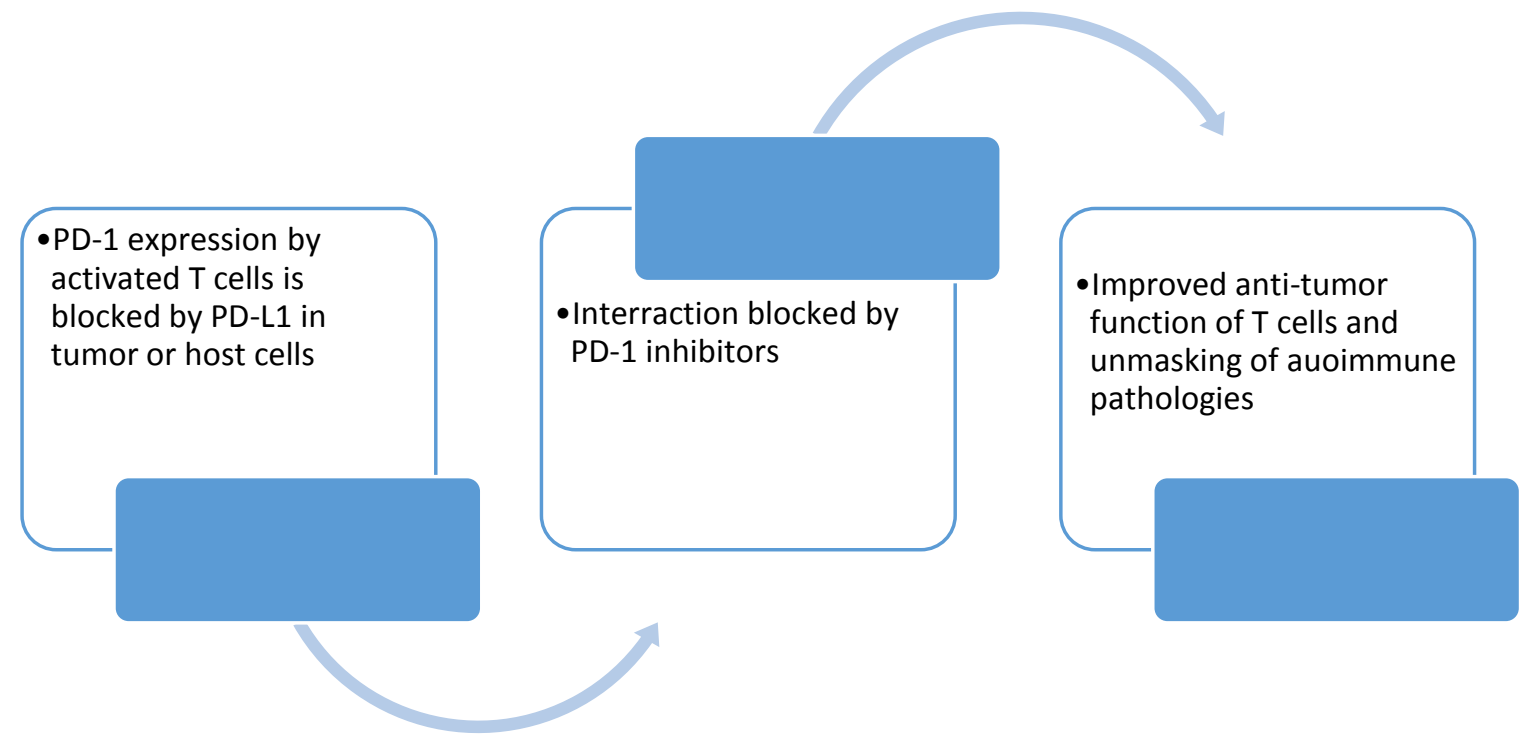

Figure 3: Mechanism of action of programmed cell death-1 (PD-1) inhibitors 
A series of checkmate trials have led to the accelerated approval of these immune check point inhibitors in the treatment of metastatic melanoma, renal cell cancer, non-small cell lung cancer, small cell cancer and urothelial cancer. ${ }^{[8-14]}$ Also, its use in the treatment of hepatocellular cancer is under an expedited review by FDA. ${ }^{[15]}$ The first CTLA-4 inhibitor in the market was ipilimumab and one of the most widely used PD-1 inhibitor is nivolumab. Both have been used with great success in the treatment of metastatic melanoma with improved survival at 5 years. ${ }^{[16-20]}$ It is also showing promise in the treatment of Hodgkin's lymphoma. ${ }^{[21]}$ With their outstanding efficacy in many cancers, their use is likely to increase in clinical practice. They are effective in younger as well as older age groups. ${ }^{[2]}$

\section{Immune mediated endocrinopathies related to the use of PD-1 inhibitors}

Common terminology criteria for adverse events (CTCAE) grades the medication related side effects from grade 1-4. Grade 1 being the mildest and grade 4 the most severe. In grade 2 side effects, daily activity is affected and minimal medical intervention is necessary. With grade 3 , marked limitation of activity happens and hospitalization may be required. Most of the PD-1 inhibitor related side effects are classified as grade 2. But if the care providers are complacent, these endocrinopathies could be missed and this could lead to grade 3-4 side effects per CTCAE criteria. Most of the endocrinopathies if recognized in time are reversible or easily managed. ${ }^{[23-30]}$

In this context, it is very important that physicians of all specialties be familiar with the immune mediated adverse events (irAEs). This would help in early identification and treatment. ${ }^{[31]}$ Common irAEs include skin rashes, hepatitis, nephritis, diarrhea / colitis, pneumonitis and endocrine dysfunction. ${ }^{[32]}$ The most common endocrine glands affected are thyroid, adrenal and pituitary. These glands are highly vascular and that could be the reason for their propensity to be affected disproportionately. There could be a clinical bias in picking these, as the side effects related to these are potentially life threatening and could easily be quantified with lab parameters.

The thyroid gland is the most common endocrine organ affected by PD-1 inhibitors. The patient may manifest with transient or long-term hypo or hyperthyroidism, thyroid eye disease, painless thyroiditis or sometimes thyroid storm. ${ }^{[33]}$ This calls for frequent monitoring and cautious use of THRT or thionamides as the case might be. ${ }^{[34]}$ Hypothyroidism is more common as was seen in our case, although the patient had an underlying subclinical hyperthyroidism. The highest grade of this side effect is usually classified as grade 2 in checkmate 057 trial, in which nivolumab was assessed in the treatment of non- squamous cell non-small cell lung cancer (NSCLC). ${ }^{[35]}$ But if the endocrinopathies are not captured and treated in time, it could quickly progress to grade 3 or 4 . This of course would require hospitalization and possibly increase mortality and morbidity associated with the use of PD-1 inhibitors. Also, the treatment of these immune related side effects includes the use of glucocorticoids. But steroids are not recommended when treating immune check point inhibitor related thyroid dysfunction. The thyroid disorders are treated in the usual manner and there is no need to interrupt the treatment with PD-1 inhibitors. ${ }^{[23,30,36,37]}$ The long-term data on treatment of PD-1 inhibitor related endocrinopathies is not there, largely because these drugs are used in metastatic malignancies and not for long periods of time.

Fulminant type 1 diabetes (DM1) has been reported with the use of PD-1 inhibitors. ${ }^{[38]}$ DM1 could present as diabetic ketoacidosis (DKA). This may lead to hospitalization and could be potentially life threating, if not picked up on time. ${ }^{[39]}$

PD-1 inhibitors are a big advancement in the armamentarium of the oncologist in their fight against metastatic malignancies. Also, timely and appropriate management of irAEs is likely to maximize the therapeutic benefit of these immune check point inhibitors. ${ }^{\left[{ }^{5,40]}\right.}$ They have been shown to be safe with respect to cardiac toxicity. The drug has been shown not to increase the QT interval. ${ }^{[41]}$ But irAEs, though benign, are relatively common with PD-1 inhibitors. It could vary from new onset vitiligo to DKA related to a new onset of DM1. Due diligence needs to be exercised before ascribing the cause of presenting pathology to these immune modulators. ${ }^{\left[{ }^{[2]}\right]}$ But if not recognized in time, these endocrinopathies could potentially present as life threatening acute emergencies. ${ }^{[33]}$ A team based approach that includes an endocrinologist could potentially help in maximizing the benefits associated with the use of immune modulating drugs in the treatment of metastatic malignancies. ${ }^{[43]}$ In our case, the patient got the first dose of nivolumab and had profound changes in thyroid function profile. This raised the question whether some patients are more at risk for endocrinopathies related to these medications and need more intense monitoring of their hormonal levels. The hospitalization in this case could have been prevented if the dramatic rise of TSH had been picked up earlier, and the patient was started on thyroid hormone replacement therapy (THRT).

\section{Potential pretreatment predictors of PD-1 inhibitor related endocrinopathies}

Evaluation of other immune check points like LAG3, TIM3 , BLTA and B7-H3 are also under evaluation and newer immune check point modulators are likely to be available in 
the years ahead. ${ }^{[4]}$ With wide spread use of immune check point inhibitors, it is becoming increasingly important to minimize the irAEs. Cancer immune therapy consortium has come out with guidelines to ensure minimal side effects and maximize benefits from these immune check point inhibitors. ${ }^{[43]}$ The crux lies in identifying patients who are at high risk for immune therapy related endocrinopathies. Potential pretreatment predictors include duration or dose of PD-1 inhibitors, preexisting autoimmune disease, race, and combination therapy with other PD-1 inhibitors. The incidence of thyroid dysfunction associated with ipilimumab is reported to be $1-6 \%$, but in combination with nivolumab it is $9.9-22 \% .^{[29,35]}$ This reinforces the need for more diligent monitoring of TFT in patients on combination therapy.

Since unfolding of the autoimmunity is the prime mechanistic explanation for endocrinopathies related to these drugs it would be prudent to get pretreatment assessment of thyroid function. This could potentially help define the pretreatment possibility of PD-1 inhibitor related endocrinopathies. This has been reported in the past. ${ }^{[5]}$ It was true in our case too as the patient had underlying subclinical hyperthyroidism. This could also help define the group of patients who are likely to have transient versus permanent endocrinopathies in the post treatment phase.

\section{CONCLUSION}

As the use of PD-1 inhibitors becomes more widespread, there is a need to identify patients who are at an increased risk of PD-1 inhibitor related endocrinopathies. Pretreatment collaboration between oncologists and endocrinologists would be helpful. This could potentially help reduce the morbidity and mortality in patients with metastatic malignancy and at the same time help reduce cost.

\section{Conflict of Interest}

The authors declare no conflict of interest.

\section{REFERENCES}

1. Bonnet C, Beinse G, Cabel L, Cochereau D, Lavaud P, Rochefort P, et al. [ESMO ECCO 2015: The highlights of immunotherapy and targeted therapies]. Bull Cancer 2016; 103: 594-603.

2. Ornstein MC, Rini BI. The safety and efficacy of nivolumab for the treatment of advanced renal cell carcinoma. Expert Rev Anticancer Ther 2016; 16: 577-84.

3. Grünwald V. Checkpoint Blockade - a New Treatment Paradigm in Renal Cell Carcinoma. Oncol Res Treat 2016; 39: 353-8.

4. Motzer RJ, Escudier B, McDermott DF, George S, Hammers HJ, Srinivas S, et al. Nivolumab versus Everolimus in Advanced Renal-Cell Carcinoma. N Engl J Med 2015; 373: 1803-13.
5. Grimm MO, Winkler Y, Fetter I, Oppel-Heuchel H. [Renaissance of immuno-oncology for urological tumors : Current status]. Urologe A 2016; 55: 621-6.

6. Buchbinder EI, Desai A. CTLA-4 and PD-1 Pathways: Similarities, Differences, and Implications of Their Inhibition. Am J Clin Oncol 2016; 39: 98-106.

7. Messerschmidt JL, Prendergast GC, Messerschmidt GL. How Cancers Escape Immune Destruction and Mechanisms of Action for the New Significantly Active Immune Therapies: Helping Nonimmunologists Decipher Recent Advances. Oncologist 2016; 21: 233-43.

8. Escudier B, Sharma P, McDermott DF, George S, Hammers HJ, Srinivas S, et al. CheckMate 025 Randomized Phase 3 Study: Outcomes by Key Baseline Factors and Prior Therapy for Nivolumab Versus Everolimus in Advanced Renal Cell Carcinoma. Eur Urol 2017; Mar. pii: S03022838(17)30099-4. [Epub ahead of print]

9. Hellmann MD, Rizvi NA, Goldman JW, Gettinger SN, Borghaei H, Brahmer JR, et al. Nivolumab plus ipilimumab as first-line treatment for advanced non-small-cell lung cancer (CheckMate 012): results of an open-label, phase 1, multicohort study. Lancet Oncol 2017; 18: 31-41.

10. Sharma P, Retz M, Siefker-Radtke A, Baron A, Necchi A, Bedke J, et al. Nivolumab in metastatic urothelial carcinoma after platinum therapy (CheckMate 275): a multicentre, single-arm, phase 2 trial. Lancet Oncol 2017; 18: 312-22.

11. Antonia SJ, López-Martin JA, Bendell J, Ott PA, Taylor M, Eder JP, et al. Nivolumab alone and nivolumab plus ipilimumab in recurrent small-cell lung cancer (CheckMate 032): a multicentre, open-label, phase 1/2 trial. Lancet Oncol 2016; 17: 883-95.

12. Cella D, Grünwald V, Nathan P, Doan J, Dastani H, Taylor F, et al. Quality of life in patients with advanced renal cell carcinoma given nivolumab versus everolimus in CheckMate 025: a randomised, open-label, phase 3 trial. Lancet Oncol 2016; 17: 994-1003.

13. Kazandjian D, Suzman DL, Blumenthal G, Mushti S, He K, Libeg M, et al. FDA Approval Summary: Nivolumab for the Treatment of Metastatic Non-Small Cell Lung Cancer with Progression On or After PlatinumBased Chemotherapy. Oncologist 2016; 21: 634-42.

14. Sharma P, Callahan MK, Bono P, Kim J, Spiliopoulou P, Calvo E, et al. Nivolumab monotherapy in recurrent metastatic urothelial carcinoma (CheckMate 032): a multicentre, open-label, two-stage, multi-arm, phase 1/2 trial. Lancet Oncol 2016; 17: 1590-8.

15. El-Khoueiry AB, Sangro B, Yau T, Crocenzi TS, Kudo M, Hsu C, et al. Nivolumab in patients with advanced hepatocellular carcinoma (CheckMate 040): an open-label, non-comparative, phase 1/2 dose escalation and expansion trial. Lancet 2017; 389: 2492-502.

16. Dramatic Survival Benefit with Nivolumab in Melanoma. Cancer Discov 2016; 6: OF7.

17. Nivolumab Doubles Survival for Patients with HNSCC. Cancer Discov 2016; 6: OF3.

18. Gupta AK, Daigle D. Nivolumab for Metastatic Melanoma. Skin Therapy Lett 2016; 21: 6-9.

19. Jin $\mathrm{C}$, Zhang $\mathrm{X}$, Zhao $\mathrm{K}, \mathrm{Xu}$ J, Zhao $\mathrm{M}, \mathrm{Xu} \mathrm{X}$. The efficacy and safety of nivolumab in the treatment of advanced melanoma: a meta-analysis of clinical trials. Onco Targets Ther 2016; 9: 1571-8.

20. Bowyer S, Prithviraj P, Lorigan P, Larkin J, McArthur G, Atkinson V, et al. Efficacy and toxicity of treatment with the anti-CTLA-4 antibody ipilimumab in patients with metastatic melanoma after prior anti-PD-1 therapy. Br J Cancer 2016; 114: 1084-9.

21. Arulogun S, Hertzberg M, Gandhi MK. Recent treatment advances in Hodgkin Lymphoma: a concise review. Intern Med J 2016; 46: 1364-9.

22. Nishijima TF, Muss HB, Shachar SS, Moschos SJ. Comparison of efficacy of immune checkpoint inhibitors (ICIs) between younger and older patients: A systematic review and meta-analysis. Cancer Treat Rev 2016; 45: 30-7.

23. Oppel-Heuchel H, Grimm MO. [Therapy monitoring and management of adverse events in PD-1/PD-L1 immune checkpoint inhibition]. Urologe 
A 2016; 55: 677-90.

24. Postow MA. Managing immune checkpoint-blocking antibody side effects. Am Soc Clin Oncol Educ Book 2015: 76-83.

25. Rizvi NA, Mazières J, Planchard D, Stinchcombe TE, Dy GK, Antonia SJ, et al. Activity and safety of nivolumab, an anti-PD-1 immune checkpoint inhibitor, for patients with advanced, refractory squamous non-small-cell lung cancer (CheckMate 063): a phase 2, single-arm trial. Lancet Oncol 2015; 16: 257-65.

26. Villadolid J, Amin A. Immune checkpoint inhibitors in clinical practice: update on management of immune-related toxicities. Transl Lung Cancer Res 2015; 4: 560-75.

27. Wehler T, Wehler B, Stehle I. [Immunotherapy in lung cancer: checkpoint inhibitors]. Dtsch Med Wochenschr 2015; 140: 1835-8.

28. O'Sullivan Coyne G, Madan RA, Gulley JL. Nivolumab: promising survival signal coupled with limited toxicity raises expectations. J Clin Oncol 2014; 32: 986-8.

29. Ryder M, Callahan M, Postow MA, Wolchok J, Fagin JA. Endocrinerelated adverse events following ipilimumab in patients with advanced melanoma: a comprehensive retrospective review from a single institution. Endocr Relat Cancer 2014; 21: 371-81.

30. Rossi E, Sgambato A, De Chiara G, Casaluce F, Losanno T, Sacco PC, et al. Endocrinopathies induced by immune-checkpoint inhibitors in advanced non-small cell lung cancer. Expert Rev Clin Pharmacol 2016; 9: 419-28.

31. Eigentler TK, Hassel JC, Berking C, Aberle J, Bachmann O, Grünwald V, et al. Diagnosis, monitoring and management of immune-related adverse drug reactions of anti-PD-1 antibody therapy. Cancer Treat Rev 2016; 45: 7-18.

32. Seetharamu N, Budman DR, Sullivan KM. Immune checkpoint inhibitors in lung cancer: past, present and future. Future Oncol 2016; 12: 1151-63.

33. McMillen B, Dhillon MS, Yong-Yow S. A rare case of thyroid storm. BMJ Case Rep 2016; 2016: 10.1136/bcr-2016-214603.

34. Verma I, Modi A, Tripathi H, Agrawal A. Nivolumab causing painless thyroiditis in a patient with adenocarcinoma of the lung. BMJ Case Rep. 2016; 2016. pii: bcr2015213692.

35. Larkin J, Chiarion-Sileni V, Gonzalez R, Grob JJ, Cowey CL, Lao CD, et al. Combined Nivolumab and Ipilimumab or Monotherapy in Untreated Melanoma. N Engl J Med. Jul 2015; 373: 23-34.
36. Sgambato A, Casaluce F, Sacco PC, Palazzolo G, Maione P, Rossi A, et al. Anti PD-1 and PDL-1 Immunotherapy in the Treatment of Advanced Non- Small Cell Lung Cancer (NSCLC): A Review on Toxicity Profile and its Management. Curr Drug Saf 2016; 11: 62-8.

37. Spain L, Diem S, Larkin J. Management of toxicities of immune checkpoint inhibitors. Cancer Treat Rev 2016; 44: 51-60.

38. Hughes J, Vudattu N, Sznol M, Gettinger S, Kluger H, Lupsa B, et al. Precipitation of autoimmune diabetes with anti-PD-1 immunotherapy. Diabetes Care 2015; 38: e55-7.

39. Okamoto M, Gotoh K, Masaki T, Masaki T, Ozeki Y, Ando H, et al. Fulminant type 1 diabetes mellitus with anti-programmed cell death-1 therapy. J Diabetes Investig 2016; 7: 915-8.

40. Kourie HR, Klastersky JA. Side-effects of checkpoint inhibitor-based combination therapy. Curr Opin Oncol 2016; 28: 306-13.

41. Agrawal S, Waxman I, Lambert A, Roy A, Darbenzio R. Evaluation of the potential for QTc prolongation in patients with solid tumors receiving nivolumab. Cancer Chemother Pharmacol 2016; 77: 635-41.

42. Carter CA, Browning R, Oronsky BT, Scicinski JJ, Brzezniak C. The Case of a Zebra That Was Misdiagnosed as a Horse: Pulmonary Tumor Thrombotic Microangiopathy, a New Paraneoplastic Syndrome, Mimicking PD-1-Induced Pneumonitis. Case Rep Oncol 2016 2016; 9: 68-75.

43. Mellman I, Hubbard-Lucey VM, Tontonoz MJ, Kalos MD, Chen DS, Allison JP, et al. De-Risking Immunotherapy: Report of a Consensus Workshop of the Cancer Immunotherapy Consortium of the Cancer Research Institute. Cancer Immunol Res 2016; 4: 279-88.

44. Swatler J, Kozłowska E. [Immune checkpoint-targeted cancer immunotherapies]. Postepy Hig Med Dosw (Online) 2016; 70: 25-42.

45. Narita T, Oiso N, Taketomo Y, Okahashi K, Yamauchi K, Sato M, et al. Serological aggravation of autoimmune thyroid disease in two cases receiving nivolumab. J Dermatol 2016; 43: 210-4.

How to cite this article: Sehgal V, Childress R. Urgent need to define Pretreatment predictors of immune check point inhibitors related endocrinopathies: A case report and review of literature. J Transl Intern Med 2017; 5: 235-9. 